(1)

CrossMark

\title{
Biomarkers in community-acquired pneumonia: still searching for the one
}

\author{
Oriol Sibila (1) ${ }^{1,2}$ and Marcos I. Restrepo ${ }^{3}$
}

Affiliations: ${ }^{1}$ Servei de Pneumologia, Hospital de la Santa Creu i Sant Pau, Barcelona, Spain. ${ }^{2}$ Institut d'Investigació Biomèdica Sant Pau (IIB Sant Pau), Barcelona, Spain. ${ }^{3}$ Division of Pulmonary Diseases and Critical Care Medicine, The University of Texas Health Science Center at San Antonio, San Antonio, TX, USA.

Correspondence: Oriol Sibila, Servei de Pneumologia, Hospital de la Santa Creu i Sant Pau, C/ Sant Antoni M. Claret 167, 08025 Barcelona, Spain. E-mail: osibiladsantpau.cat

@ERSpublications

Fibroblast growth factor 21 (FGF21) predicts severity of illness, clinical stability and mortality in community-acquired pneumonia. Validation is needed to confirm the application of FGF21 in clinical practice. http://ow.ly/SYI730nuRc1

Cite this article as: Sibila O, Restrepo MI. Biomarkers in community-acquired pneumonia: still searching for the one. Eur Respir J 2019; 53: 1802469 [https://doi.org/10.1183/13993003.02469-2018].

Community-acquired pneumonia (CAP) remains a major cause of morbidity and mortality worldwide [1]. Despite advances in antibiotic treatment and medical care, the mortality of CAP is still high in hospitalised patients, especially in those with severe illness [2]. Appropriate initial severity assessment is a crucial step in pneumonia management, since it has been demonstrated that an early recognition of severe CAP patients improves their clinical outcomes [3]. Several tools have been developed to evaluate disease severity, in particular focusing on predicting hospital admission and mortality [4]. However, recent studies have showed that most of these scores are not used routinely in clinical practice and may be inadequate tools to guide appropriate antibiotic treatment $[5,6]$.

Biomarkers are biological markers that can be measured accurately and reproducibly from patients' samples. In pneumonia, several studies have evaluated the role of biomarkers in different important clinical areas, such as diagnosis of pneumonia, aetiology, risk stratification and triage decision, severity of illness and initiation/duration/discontinuation of antibiotic therapy [7]. Most of these studies focused on inflammatory markers that reflect the interaction between the micro-organism and the host, which is crucial in CAP prognosis and severity of disease [8]. The two most common inflammatory biomarkers are C-reactive protein (CRP) and procalcitonin (PCT). However, other biomarkers with less available evidence have been tested for a wide range of clinical outcomes, and include sTREM (soluble triggering receptor expressed on myeloid cells) $[9,10]$, pro-adrenomedullin $[11,12]$, pro-atrial natriuretic peptide $[13,14]$, pro-vasopressin $[15,16]$, surfactant protein-D, human cartilage glycoprotein YKL-40 and chemokine ligand 18 [17], endocan [18], barrier-stabilising angiopoietins 1 and 2 [19], or markers of neutrophil extracellular traps [20]. Figure 1 summarises the most common biomarkers evaluated and their potential role in assessing different clinical outcomes in patients with CAP.

In this issue of European Respiratory Journal, EBRAнIмI et al. [21] studied the prognostic value in CAP patients of fibroblast growth factor 21 (FGF21), a peptide hormone that has been shown to act as a metabolite regulator of glucose homeostasis, ketogenesis, insulin sensitivity and lipid metabolism [22]. Several organs synthesise FGF21, including the liver, brown adipose tissue, inguinal white adipose tissue, gonadal white adipose tissue, muscle, pancreas and heart. Therefore, it can act on multiple target tissues in either a paracrine or an endocrine fashion. It is postulated that FGF21 may play an important role in the 


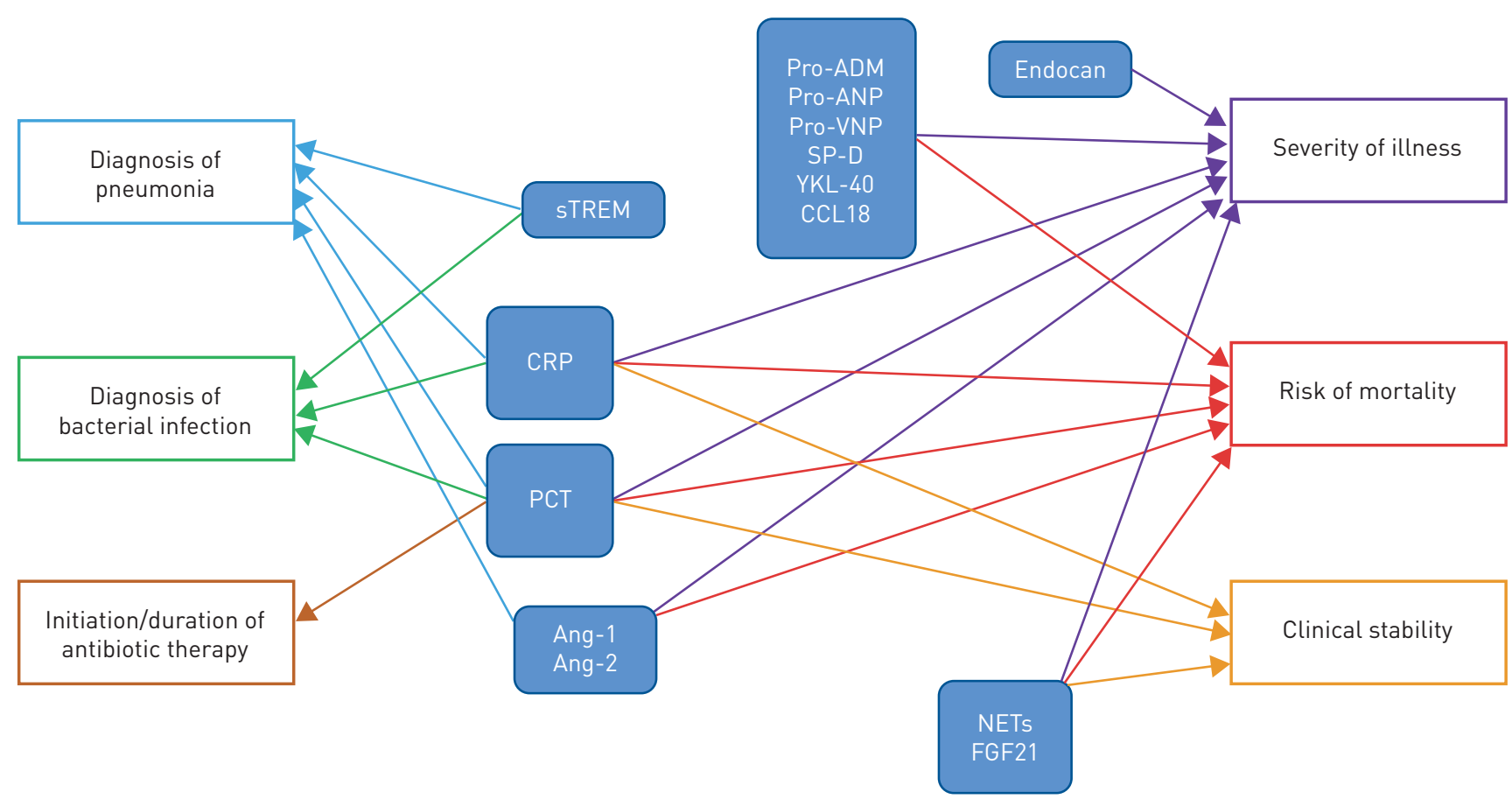

FIGURE 1 Inflammatory biomarkers and their relationship with community-acquired pneumonia outcomes most studied in the past 10 years. STREM: soluble triggering receptor expressed on myeloid cells; CRP: C-reactive protein; PCT: procalcitonin; Ang: barrier-stabilising angiopoietin; pro-ADM: pro-adrenomedullin; pro-ANP: pro-atrial natriuretic peptide; pro-VNP: pro-vasopressin; SP-D: surfactant protein-D; YKL-40: human cartilage glycoprotein YKL-40; CCL18: chemokine ligand 18; NET: neutrophil extracellular trap.

immunoregulation of pro- and anti-inflammatory mediators in systemic inflammatory states such as pneumonia. Using samples and clinical data of two previous well-defined cohorts of hospitalised CAP patients, the authors found that FGF21 levels at admission were related to disease severity and identify patients at risk of 30-day all-cause mortality with superior discriminate power compared to than routine markers such as CRP, PCT or white cell count. The prognostic performance of FGF21 for the prediction of 30-day mortality was better than commonly used clinical scores such as CURB-65 and comparable to the Pneumonia Severity Index (PSI). In addition, adjunct treatment with corticosteroids, which potentially improved clinical outcomes in these patients [23], led to decreasing FGF21 levels, which is related to clinical stability. These findings are novel and suggest that the determination of FGF21 on admission and during hospitalisation might provide information regarding the severity of CAP, time to clinical stability and risk of short-term mortality.

However, it is important to remark that the use of FGF21 as a CAP biomarker in clinical practice is still undermined by many uncertainties and questions. First, it is unclear the equipment that would be needed and the cost related to measure FGF21. This may bring difficulties with implementation in a "real world scenario" compared to the easily accessible and cost-effective. Second, the identification of clinically relevant cut-off values is critical in the interpretation and adoption of this new biomarker. Third, as seen with corticosteroids, comorbid conditions or other concomitant treatments may potentially affect FGF21 levels. And finally, validation studies are needed in order to prove that FGF21 is here to compete against other biomarkers, before final adoption in clinical practice.

In summary, FGF21 is a novel biomarker tested to predict CAP severity. However, time and new evidence will tell whether FGF21 will become a biomarker that will assist clinicians in decision-making regarding care for patients with CAP, and to help improve patients' clinical outcomes. It is encouraging to see a growing trend in identifying new molecules and novel biomarkers for a neglected disease that affects millions of patients around the world [24].

Conflict of interest: None declared.

\section{References}

1 Waterer G. Community-acquired pneumonia: a global perspective. Semin Respir Crit Care Med 2016; 37: 799-805.

2 Kolditz M, Braeken D, Ewig S, et al. Severity assessment and the immediate and long-term prognosis in community-acquired pneumonia. Semin Respir Crit Care Med 2016; 37: 886-896. 
3 Sibila O, Restrepo MI, Anzueto A. What is the best antimicrobial treatment for severe community-acquired pneumonia (including the role of steroids and statins and other immunomodulatory agents). Infect Dis Clin North Am 2013; 27: 133-147.

4 Fernandez JF, Sibila O, Restrepo MI. Predicting ICU admission in community-acquired pneumonia: clinical scores and biomarkers. Expert Rev Clin Pharmacol 2012; 5: 445-458.

5 Serisier DJ, Williams S, Bowler SD. Australasian respiratory and emergency physicians do not use the pneumonia severity index in community-acquired pneumonia. Respirology 2013; 18: 291-296.

6 Singanayagam A, Aliberti S, Cillóniz C, et al. Evaluation of severity score-guided approaches to macrolide use in community-acquired pneumonia. Eur Respir J 2017; 50: 1602306.

7 Sungurlu S, Balk RA. The role of biomarkers in the diagnosis and management of pneumonia. Clin Chest Med 2018; 39: 691-701.

8 Guertler C, Wirz B, Christ-Crain $\mathrm{M}$, et al. Inflammatory responses predict long-term mortality risk in community-acquired pneumonia. Eur Respir J 2011; 37: 1439-1446.

9 Gibot S, Cravoisy A, Levy B, et al. Soluble triggering receptor expressed on myeloid cells and the diagnosis of pneumonia. N Engl J Med 2004; 350: 451-458.

10 Esposito S, Di Gangi M, Cardinale F, et al. Sensitivity and specificity of soluble triggering receptor expressed on myeloid cells-1, midregional proatrial natriuretic peptide and midregional proadrenomedullin for distinguishing etiology and to assess severity in community-acquired pneumonia. PLoS One 2016; 11: e0163262.

11 Krüger S, Ewig S, Giersdorf S, et al. Cardiovascular and inflammatory biomarkers to predict short- and long-term survival in community-acquired pneumonia. Am J Respir Crit Care Med 2010; 182: 1426-1434.

12 Bello S, Lasierra $\mathrm{AB}$, Mincholé $\mathrm{E}$, et al. Prognostic power of proadrenomedullin in community-acquired pneumonia is independent of aetiology. Eur Respir J 2012; 39: 1144-1155.

13 Müller B, Süess E, Schuetz P, et al. Circulating levels of pro-atrial natriuretic peptide in lower respiratory tract infections. J Intern Med 2006; 260: 568-576.

14 Ramírez P, Ferrer M, Martí V, et al. Inflammatory biomarkers and prediction for intensive care unit admission in severe community-acquired pneumonia. Crit Care Med 2011; 39: 2211-2217.

15 Masiá M, Papassotiriou J, Morgenthaler NG, et al. Midregional pro-A-type natriuretic peptide and carboxy-terminal provasopressin may predict prognosis in community-acquired pneumonia. Clin Chem 2007; 53: 2193-2201.

16 Müller B, Morgenthaler N, Stolz D, et al. Circulating levels of copeptin, a novel biomarker, in lower respiratory tract infections. Eur J Clin Invest 2007; 37: 145-152.

17 Spoorenberg SMC, Vestjens SMT, Rijkers GT, et al. YKL-40, CCL18 and SP-D predict mortality in patients hospitalized with community-acquired pneumonia. Respirology 2017; 22: 542-550.

18 Mangat M, Amalakuhan B, Habib S, et al. High endocan levels are associated with the need for mechanical ventilation among patients with severe sepsis. Eur Respir J 2017; 50: 1700013.

19 Gutbier B, Neuhauß A-K, Reppe K, et al. Prognostic and pathogenic role of angiopoietin-1 and -2 in pneumonia. Am J Respir Crit Care Med 2018; 198: 220-231.

20 Ebrahimi F, Giaglis S, Hahn S, et al. Markers of neutrophil extracellular traps predict adverse outcome in community-acquired pneumonia: secondary analysis of a randomised controlled trial. Eur Respir J 2018; 51: 1701389 .

21 Ebrahimi F, Wolffenbuttel C, Blum CA, et al. Fibroblast growth factor 21 predicts outcome in community-acquired pneumonia: secondary analysis of two randomised controlled trials. Eur Respir J 2019; 53 1800973.

22 Fisher FM, Maratos-Flier E. Understanding the physiology of FGF21. Annu Rev Physiol 2016; 78: 223-241.

23 Sibila O, Rodrigo-Troyano A, Torres A. Nonantibiotic adjunctive therapies for community-acquired pneumonia (corticosteroids and beyond): where are we with them? Semin Respir Crit Care Med 2016; 37: 913-922.

24 Aliberti S, Dela Cruz CS, Sotgiu G, et al. Pneumonia is a neglected problem: it is now time to act. Lancet Respir Med 2019; 7: 10-11. 